\title{
Patterns of Interpersonal Problems Associated with Binge Eating D isorder
}

\author{
Kathleen L. Eldredge, ${ }^{1 *}$ Kenneth D. Locke, ${ }^{2}$ and Leonard M. Horowitz ${ }^{3}$ \\ ${ }^{1}$ Department of Psychiatry and Behavioral Sciences, Stanford University School of \\ Medicine, Stanford, California \\ ${ }^{2}$ Department of Psychology, University of Idaho, Moscow, Idaho \\ ${ }^{3}$ Department of Psychology, Stanford University, Stanford, California
}

Accepted 20 November 1996

\begin{abstract}
Absract: 0 bjective: To determine if interpersonal problems reported by individuals with binge eating disorder (BED) are distinct from psychiatric norms, and whether specific types of interpersonal problems are predictive of BED treatment outcome. Method: Subjects were 88 females with BED who completed a treatment trial for binge eating and weight loss. Pretreatment data on the Inventory of Interpersonal Problems (IIP) was compared (1) to that of psychiatric and nonpsychiatric norms and (2) for individuals with good versus poor outcome for eating disorder symptoms and weight loss. Results: BED patients reported less distress for problems with being too vindictive (hostile dominance) than psychiatric norms. Patients with good eating disorder outcome reported less distress for problems with social avoidance; patients with good weight loss outcome reported greater distress over problems with vindictiveness. Discussion: BED treatment may be enhanced by an initial focus on specific interpersonal difficulties. (c) 1998 by John Wiley \& Sons, Inc. Int J Eat Disord 23: 383-389, 1998.
\end{abstract}

Key words: binge eating disorder; interpersonal problems; psychiatric norms

\section{INTRO DUCTIO N}

Little is known about the relationship between interpersonal problems and binge eating disorder (BED). Research to date indicates that interpersonal problems are significantly correlated with severity of binge eating but not overweight (Telch \& Agras, 1994); that factors related to interpersonal relationships, mood, and thoughts are more influential in precipitating binge eating in obese binge eaters than physiological factors (Arnow, Kenardy, \& Agras, 1992); and that individuals with BED report a higher prevalence of impaired relations with people and work impairments due to upset about weight and

\footnotetext{
${ }^{*}$ Correspondence to: Dr. Kathleen Eldredge, Behavioral Medicine Program, Department of Psychiatry, Stanford University School of Medicine, Stanford, CA 94305.

(C) 1998 by John Wiley \& Sons, Inc.

CCC 0276-3478/98/040383-07
} 
eating than the nonbinging obese (Spitzer et al., 1993). Studies of the prevalence of personality disorders among individuals with BED as compared to subclinical or nonbinge eating obese individuals indicate individuals with BED to have a higher lifetime prevalence of borderline and avoidant personality disorders (Yanovski, Nelson, Dubbert, \& Spitzer, 1993) and to have more frequent current diagnoses of histrionic, borderline, and avoidant personality disorders (Specker, de Zwaan, Raymond, \& Mitchell, 1994). The research thus suggests that BED is associated with interpersonal distress.

To date, research has not specified the types of interpersonal problems most frequently experienced by individuals with BED, nor whether the interpersonal problems experienced by individuals with BED differ from the types of interpersonal distress and impaired relationships seen in psychiatric patients in general. A useful model for describing the nature of interpersonal problems is the interpersonal circumplex. This model claims that interpersonal behaviors in general can be organized graphically in two dimensions. One dimension, a dimension of affiliation, nurturance, or communion, ranges from friendly or warm behavior to hostile or cold behavior. The other dimension, a dimension of control, dominance, or influence, ranges from dominating or controlling behavior to yielding or relinquishing control. Thus, interpersonal problems exist that correspond to every combination of the two underlying factors: Some problems reflect too much friendliness (or too much hostility); others reflect too much dominance (or too much submissiveness). (See Alden, Wiggins, \& Pincus, 1990, for a more complete description of the circumplex).

The first aim of our study was to use the interpersonal circumplex to determine if the pattern of interpersonal problems reported by individuals with BED is distinct from that reported by other psychiatric patients. The second aim was to determine whether specific types of interpersonal problems would be predictive of treatment outcome for individuals with BED. Treatment in our study consisted of 9 months of behavioral and/or cognitivebehavioral therapy. We assessed both interpersonal problems and eating-related outcome variables prior to, during, and after treatment. We were able to examine whether interpersonal problems prior to treatment would predict response to treatment; and, specifically, to treatment focused on eating-related, rather than interpersonal, cognitions and behaviors.

\section{METHOD}

\section{O verview}

Subjects meeting proposed diagnostic criteria for BED completed a measure of interpersonal problems, the Inventory of Interpersonal Problems (IIP; Horowitz, Rosenberg, Baer, Ureno, \& Villasenor, 1988) prior to their participation in a 36-week treatment trial for BED. The treatment trial was designed to compare the effects of cognitive-behavioral treatment, weight loss treatment, and desipramine on binge eating and weight loss. Subjects received either 9 months of weight loss only treatment; 3 months of cognitivebehavioral treatment followed by weight loss treatment for 6 months; or the latter treatment with desipramine added for the final 6 months. Subjects' scores on the IIP were compared to those from a general (primarily female) outpatient psychiatric population comparable in age, education, and ethnicity, but more diverse in the nature of the pa- 
tients' complaints. Scoring profiles on the IIP of subjects who succeeded versus failed at treatment were also compared.

\section{Subjects}

The original sample consisted of 108 treated female subjects (see Agras et al., 1995, for an overview of the study and subject selection criteria). However, only those subjects who completed the 36 weeks of treatment (defined as having been weighed at both baseline and termination; $n=88$ ) were included in the current study. The number of subjects included in different analyses vary because some subjects did not complete all the relevant measures at each time period.

The women in the subject sample were primarily Caucasian $(90.9 \%)$, with Blacks (3.4\%), Hispanics (3.4\%), and Other (2.3\%) composing the remainder of the sample. The majority of subjects were employed $(80.7 \%)$, and the sample as a whole was well educated $(38.6 \%$ attended some college; $21.6 \%$ completed college; $31.8 \%$ completed graduate or professional school). Mean age of the sample was 44.7 years $(S D=10.8)$, while the mean weight of subjects at baseline was $228.8 \mathrm{lb}(S D=38.0)$ and the mean age of onset of binge eating was 18.4 years $(S D=10.4)$.

\section{Measures}

IIP

The measure of interpersonal problems was the IIP (Horowitz et al., 1988), a self-report inventory designed to assess a range of maladaptive interpersonal dispositions. The eight subscales of the IIP (too Domineering, Vindictive, Cold, Socially Avoidant, Nonassertive, Exploitable, Overly Nurturant, Intrusive) correspond to the eight octants of the interpersonal circumplex. Raw octant scores were computed as the sum of the eight items.

In addition to computing raw octant scores, we also computed ipsative octant scores, using each patient's own mean $(M)$ and standard deviation $(S D)$ across all octant scores: (octant score - M)/SD). Because ipsative scores reflect deviations from a subject's own mean, they eliminate variance due to differences in the subjects' overall level of distress. Instead, an ipsative score shows whether a particular subscale score reflects higher-thanaverage or lower-than-average distress for that subject. We will conservatively report only those findings that are consistent across both raw and ipsative octant scores.

\section{Treatment 0 utcome}

Two types of outcome measures were used: a composite eating disorder measure and actual weight. The eating disorder measure included binge days, defined as the number of self-reported days upon which at least one binge episode occurred over a continuous 2-week period; the Restraint (scored inversely), Disinhibition, and Hunger subscale scores of the Three-Factor Eating Questionnaire (TFEQ; Stunkard \& Messick, 1985); and the Binge Eating Scale score (BES; Gormally, Black, Daston \& Rardin, 1982). At termination, the eating disorder measures were significantly correlated with each other [mean $r(80)=$ $.47, p<.01]$, but were not significantly correlated with actual weight [mean $r(80)=.20$, n.s.]. Therefore, we employed two different measures of treatment outcome: (1) eating disorder outcome (computed as the summed $\mathrm{z}$ scores of the five eating disorder measures completed at 36 weeks; alpha $=.81)$ and $(2)$ change in weight from baseline to termination. 


\section{RESU LTS}

\section{Interpersonal Characteristics of the Sample}

\section{Comparison with Psychiatric Norms}

$T$ tests comparing the mean baseline IIP octant scores (both raw and ipsative) for our BED sample $(n=87)$ and a normative psychiatric sample $(n=200)$ described in Horowitz et al. (1988) revealed significant differences for problems with being vindictive. With respect to both raw and ipsative distress scores, BED patients reported being significantly less distressed by problems with being vindictive [raw, $t(285)=2.50, p=0.13$; ipsative, $t(285)=3.50, p<.001]$. Thus, compared to a general sample of outpatients, the BED patients tended to underreport problems of vindictiveness; that is, they report less hostile dominance (fighting, criticizing) than normative psychiatric outpatients.

In order to conclude that BED patients have a characteristic profile of interpersonal problems, we wanted to demonstrate that the pattern of responding in a sample of BED patients is more homogeneous than the pattern of responding in a sample of general psychiatric patients. Therefore, we conducted a $t$ test comparing the standard deviations of the ipsative octant scores from the BED sample versus the general psychiatric sample. The results confirmed that the BED sample was indeed more homogeneous, $t(7)=2.25, p$ $<.03$, one tailed.

\section{Changes in Interpersonal Problems over Time}

To examine changes in interpersonal problems over the course of treatment, we performed repeated measures analyses of variance (ANOVAs) on subjects' self-ratings in each of the IIP octants, with time of measurement (baseline, 12 weeks, 24 weeks, and termination) as a repeated measures variable. The results showed that for every type of interpersonal problem, distress decreased over the course of treatment, all $p \mathrm{~s}<.01$. The interaction of time and octant was not significant.

\section{Comparison of Patients with Good Versus Poor O utcomes}

The next question was whether the observed patterns of interpersonal distress would differ for patients who differed in treatment outcome. We employed two measures of treatment outcome: self-reported eating disorder outcomes and change in weight. For both measures, subjects above the median were labeled "good outcomes" and subjects below the median were labeled "poor outcomes."

\section{Comparison of IIP Scores of Patients with Good Versus Poor Eating 0 utcomes}

To examine whether IIP scores differed for patients differing in self-reported eating outcomes, multivariate analyses of variance (MANOVAs) were performed on subjects' self-descriptions on each of the IIP octants, with self-reported eating outcomes (good outcome vs. poor outcome) as a between-subjects variable and time of measurement (baseline, 12 weeks, 24 weeks, and termination) as a within-subjects variable.

Across raw and ipsative scores, a main effect of self-reported outcome was found for problems with being too socially avoidant [raw, $F(1,76)=8.62, p=.004$; ipsative $[F(1,76)$ $=4.23, p=.043]$. Patients who had a good outcome tended to report fewer problems with being socially avoidant throughout treatment. 


\section{Comparison of IIP Scores of Patients with Good Versus Poor W eight $\mathbf{O}$ utcomes}

When the above analyses were repeated with subjects grouped according to changes in actual weight, a significant effect was found for the interaction of outcome and time of measurement on vindictive problems [raw, $F(3,83)=4.17, p=.007$; ipsative, $F(3,83)=2.58$, $p=.054]$. At the start of treatment, patients with good weight outcomes tended to report more problems with being vindictive than did patients with poor weight outcomes; however, this difference had disappeared by termination. Poor outcome patients had acknowledged such little distress over vindictiveness $(M=.58$ on a $0-4$ scale) that they had no room for improvement.

The pretreatment difference between good and poor outcome patients for problems with vindictiveness suggested the possibility that the low scores of the poor outcome patients were responsible for the earlier reported difference between psychiatric norms and eating disorder patients on this variable. Subsequent $t$ tests between psychiatric norms and good and poor outcome patients indicated this to be the case: good outcome versus psychiatric norms: raw, $t(2,42)=.55$, n.s.; ipsative; $t(2,42)=1.46$, n.s.; poor outcome versus psychiatric norms: raw, $t(242)=3.25, p=.001$; ipsative, $t(241)=3.88, p<.001$.

\section{DISCUSSIO N}

The primary aim of this study was to determine if the pattern of interpersonal problems reported by individuals with BED is distinct from that reported by other psychiatric patients. Our results demonstrate that individuals with BED as a group display characteristic interpersonal difficulties, as indicated by the overall consistency (i.e., the mean standard deviation across all octants) in scoring for BED patients on the IIP, and further that BED patients tend to report being less distressed by problems of vindictiveness than psychiatric norms.

The secondary aim of this study was to determine whether specific types of interpersonal problems would be predictive of treatment outcome (i.e., improvement in eating disorder or weight loss) for individuals with BED. Our findings for eating disorder outcome indicate that patients with good outcome reported significantly less distress over problems with social avoidance than patients with poor outcome. This finding is interesting in light of recent research indicating higher current (Specker et al., 1994) and lifetime (Yanovski et al., 1993) diagnoses of avoidant personality disorder among individuals with BED as compared to obese nonbingers and subclinical bingers. Our comparison of BED patients with good versus poor outcome for weight loss indicated that patients with good weight loss outcomes reported greater distress and concern over problems with being too vindictive at the beginning of treatment; however, these differences disappeared by termination. Thus, combining eating disorder and weight loss outcomes, poor outcome was associated with more distress due to problems of social avoidance and unusually low distress due to problems of vindictiveness. This suggests that individuals with poor outcome are apt to manage their feelings of hostility in a passive or submissive manner (e.g., by refusing to acknowledge them).

Why might patients who report more social avoidance (hostile submission) or who deny vindictiveness (hostile dominance) be poor bets for treatment? One possibility is that successful treatment for binge eating and weight loss in the obese calls for both behavioral and attitudinal changes. Individuals need to make specific changes not only in their eating and exercise behaviors, but also in confronting many very inflammatory personal and cultural assumptions and expectations which can defeat treatment (e.g., that obesity is a 
moral failing, that change is easy for those with strength of character). Patients who report responding to hostility in passive and submissive ways versus active and dominant ways may have a harder time assuming such a confrontational (hostile dominant) stance.

The pattern of results for social avoidance and vindictiveness was consistent across raw and ipsative scores. Methodologically, therefore, these results do not appear to reflect mere social desirability response sets; the good eating disorder outcome group and poor weight loss outcome group each showed low levels of problems (i.e., with social avoidance and vindictiveness, respectively), both in absolute terms (as evidenced by their raw scores) and relative to their overall tendency to report interpersonal problems (as evidenced by their ipsative scores). Further, the finding for the poor weight loss outcome group likely explains the previous result that individuals with BED are less distressed by problems with being too vindictive than psychiatric norms (i.e., the poor outcome subjects carried this finding).

In order to understand the potential significance of the pattern of low scores on the vindictiveness problem domain for individuals with BED relative to the psychiatric population, we were interested in comparing BED individuals with poor weight loss outcome to a normal population. For this purpose, we had available to us data from a sample of 514 college students (Alden et al., 1990). $T$ tests again showed the poor weight loss outcome subjects to score significantly lower on the vindictiveness problem domain than students: for raw scores, $t(255)=3.87, p<.001$; for ipsative scores, $t(255)=4.85, p<.001$. In other words, even for a student sample, it is normal to report a higher level of interpersonal problems related to being too vindictive than that reported by the BED individuals with poor weight outcomes. Thus, the response pattern of our poor weight outcome group suggests a remarkable paucity of reported problems in this area.

Taken together, the self-report data of our patients suggest that treatment of BED might be enhanced by addressing patients' problems with social avoidance as well as their reported avoidance of vindictiveness. An interesting question arises, however, as to whether patients' reported levels of problems with vindictiveness are accurate. If this is not the case, then this would suggest that the more appropriate target of intervention may be their difficulty acknowledging (and perhaps coping with) issues related to vindictiveness. This in turn would likely pose a particularly challenging therapeutic task, for it would call on the therapist to succeed at addressing a problem area which patients do not perceive to be of relevance to them.

\section{REFEREN CES}

Agras, W. S., Telch, C. F., Arnow, B., Eldredge, K., Detzer, M. J., Henderson, J., \& Marnell, M. (1995). Does interpersonal therapy help patients with binge eating disorder who fail to respond to cognitive-behavioral therapy? Journal of Consulting and Clinical Psychology, 63, 356-360.

Alden, L. E., Wiggins, J. S., \& Pincus, A. L. (1990). Construction of circumplex scales for the Inventory of Interpersonal Problems. Journal of Personality Assessment, 55, 521-536.

Arnow, B., Kenardy, J., \& Agras, W. S. (1992). Binge eating among the obese: A descriptive study. Journal of Behavioral Medicine, 15, 155-170.

Gormally, J., Black, S., Daston, S., \& Rardin, D. (1982). The assessment of binge eating severity among obese subjects. Addictive Behaviors, 7, 47-55.

Horowitz, L. M., Rosenberg, S. E., Baer, B. A., Ureno, G., \& Villasenor, V. S. (1988). Inventory of Interpersonal Problems: Psychometric properties and clinical applications. Journal of Consulting and Clinical Psychology, 56, 885-892.

Specker, S., de Zwaan, M., Raymond, N., \& Mitchell, J. (1994). Psychopathology in subgroups of obese women with and without binge eating disorder. Comprehensive Psychiatry, 35, 185-190.

Spitzer, R. L., Yanovski, S., Wadden, T., Wing, R., Marcus, M. D., Stunkard, A., Devlin, M., Mitchell, J., Hasin, 
D., \& Horne, R. L. (1993). Binge eating disorder: Its further validation in a multisite study. International Journal of Eating Disorders, 13, 137-153.

Stunkard, A. J., \& Messick, S. (1985). The Three-Factor Eating Questionnaire to measure dietary restraint, disinhibition, and hunger. Journal of Psychosomatic Research, 29, 71-83.

Telch, C. F., \& Agras, W. F. (1994). Obesity, binge eating and psychopathology: Are they related? International Journal of Eating Disorders, 15, 53-61.

Yanovski, S. Z., Nelson, J. E., Dubbert, B. K., \& Spitzer, R. L. (1993). Association of binge eating and psychiatric comorbidity in the obese. American Journal of Psychiatry, 150, 1472-1479. 